\section{Interbed multiples modeling for OBN data}

Claudio Guerra and Nelson Hargreaves, Petrobras

Copyright 2021, SBGf - Sociedade Brasileira de Geofísica

This paper was prepared for presentation during the $17^{\text {th }}$ International Congress of the Brazilian Geophysical Society held in Rio de Janeiro, Brazil, 16-19 August 2021.

Contents of this paper were reviewed by the Technical Committee of the $17^{\text {th }}$ International Congress of the Brazilian Geophysical Society and do not necessarily represent any position of the SBGf, its officers or members. Electronic reproduction or storage of any part of this paper for commercial purposes without the written consent of the Brazilian Geophysical Society is prohibited.

\section{Abstract}

In this work, we show an extension of the interbedmultiple modeling of Pica and Delmas (2008) for OBN data, both in the upgoing and in the downgoing mode. It uses Born modeling with the one-way wave equation. A clear advantage over other methods is that it is not doomed by the poor receiver sampling, which is characteristic in this kind of acquisition. Nonetheless, a priori information is needed, as the velocity model and the horizon in depth corresponding to the generating reflector, which, unfortunately, prevents modeling multiples generated at an unknown generator. We describe the theory with details of the method and show promising results on real OBN data of the Santos Basin.

\section{Introduction}

Interbed multiples are, by far, the most important noise interfering with the reflections from the Pre-salt reservoirs of the Santos Basin. This interference is more pronounced in the presence of the so-called stratified salt, which is mainly composed by a sequence of halite interbedded with anhydrite, and can have a huge impact when determining volumetrics, since it affects facies classification, impedance inversion, as well as, sometimes, structural interpretation. In the Santos Basin, generation of interbed multiples mainly occurs at the sea bottom and on the top salt.

Examples of successful interbed multiple attenuation in the Santos Basin include different modeling methods (Hembd et al. 2010, Cypriano et al. 2015, Krugger et al. 2018, Starring et al. 2018), all of them applied to streamer data. Some of them are data driven, while others depend on an Earth model.

Recently, Petrobras announced an aggressive node surveys campaign of nearly $18,000 \mathrm{~km}^{2}$ to be shot between 2020 and 2025 to cope with: 1) the need for wide azimuthal distribution to better image the PreSalt reservoirs; 2) the environmental restrictions on having more than one source boat firing at a distance less than $60 \mathrm{~km}$ apart, as in WAZ surveys; and 3) 4D repeatability issues for the stiff PreSalt carbonate reservoirs, for which an NRMS as low as $3 \%$ is necessary to reveal the 4D signal. Specially on this last subject, on a particular 4D node-to-node project, NRMS dropped from $2.54 \%$ after warping to $2.23 \%$ after interbed multiple attenuation $(12 \%$ drop). Therefore, for a successful reservoir monitoring with 4D in the Santos Basin, it becomes mandatory to incorporate interbed multiple attenuation for nodes acquisition geometry in the processing sequence.

Figure 1 exemplifies how misleading interbed multiples can be when interfering with the Pre-salt reflectors. Steep dipping events are signal, representing periodic lava flows of the igneous basement of the Santos Basin. Gentler dip events are interbed multiples. More misleading than this example is when interbed multiples have dips like those of the Pre-salt reflectors. This can lead interbed multiples to be interpreted as unconformities and even DHI's, which can negatively affect exploratory targets.

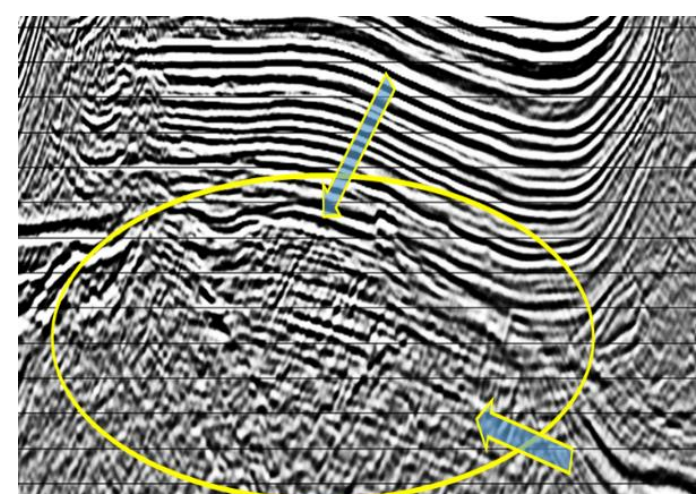

Figure 1- Steeper dip events are signal and represent periodic lava flows of the igneous basement of the Santos Basin. Gentler dip events are interbed multiples (from Guerra and Hargreaves, 2019).

Current techniques for data-driven interbed multiple attenuation for node data need an auxiliary streamer dataset to be convolved and cross-correlated with the nodes data. This imposes limitations to the result due to the limited azimuthal distribution of the streamer data. Nevertheless, successful attenuation of interbed multiples has been reported (Pereira et al., 2019)

Despite the technique, if it is data or model driven, additional information about the generator must be supplied in the form of horizons to prevent primary reflection leakage to the interbed multiple models.

Here, we adapt the method of Pica and Delmas (2008) to the OBN geometry, leveraging the experience of having previously implemented it for streamers and, additionally, in the poststack migrated domain (Guerra and Hargreaves, 2019). We show examples of real data application in the Santos Basin.

\section{Method}

Pica and Delmas (2008) describe a method of modeling interbed multiples under the $1^{\text {st }}$ order-Born approximation, in which a muted version of the data is injected to propagation subjected to a macro velocity model and convolved with a muted version of the reflectivity. Those mutes are related to the interbed-multiple generating 
reflector which, of course, must be identified. The reason behind muting is to prevent modeling of primaries in the process. Recalling the cross-correlation imaging condition

$$
m=D^{*} U,
$$

where $m$ is the estimate of the reflectivity, $D$ and $U$ are the source and receiver wavefields, respectively, and the asterisk stands for the complex-conjugate. The adjoint of the cross-correlation imaging condition is a two-sided operation,

$$
\begin{aligned}
& U=D m, \text { and } \\
& D=U m .
\end{aligned}
$$

The first adjoint operation is clearly recognized as the convolution of the source wavefield with the reflectivity, yielding the scattered wavefield, ultimately recorded at the acquisition geometry. The second adjoint operation generates the source wavefield by convolving the receiver wavefield with the reflectivity. In the process of generating the interbed multiples, this convolution is spurious since it generates a wavefield kinematically similar to the source wavefield at the depth of the generating reflector. Consequently, if not adequately handled, migration of the modeled interbed multiples will show events mimicking primary reflectors. By muting the input data at times slightly greater than that of the generating reflection prevents the so-called primary leakage to the multiple model.

Figure 2 illustrates this issue. In Figure2a is represented the migration of the downgoing wavefield of the Lula Pilot nodes base survey. On the left and on the right of the black box it is clear the interference of the interbed multiples with the PreSalt reflectors. Figure $2 b$ shows the migration of downgoing interbed multiples generated at the top salt, with a not so deep enough muted input gathers. It is clear in the black box the leakage of base of salt reflector, despite the good prediction of the interbed multiples to the left and to the right of the black box. Finally, in Figure 2c, primary leakage has been almost completely mitigated by using an adequate mute, which prevented the reflection from the top salt to convolve with the top salt reflector. It remains, however, low frequency and low amplitude events. A proper processing of these low frequency events and a specialized adaptive subtraction are key to successfully attenuate multiple without harming primary reflectors.

The estimate of the reflectivity is the migrated volume, alternatively after vertical resolution enhancement to attenuate residual wavelet and, sometimes, amplitude equalization. Input data must have gone through all noise and designature treatments and attenuation of the surface multiples. In nodes mode, it is desirable to interpolate data in the receiver domain, since shots are, in general, $50 \mathrm{~m}$ apart. we use the Reciprocity Principle to model multiples in the receiver domain, since this is the better sampled domain of nodes acquisition.

The aforementioned mute function is computed with the aid of the Born modeling of an auxiliary reflector (the generating reflector plus a depth shift), at which muting in the reflectivity is performed, by depth extrapolation and automatic picking of the maximum amplitude on each modeled trace. This more elaborated way of computing the mute function has proven to be of great importance in areas of complex top salt.

After muting and given a frequency $\omega$, data $d_{o}$ is downward extrapolated up to the maximum depth of an auxiliary horizon, few meters below the internal multiple generating reflector, according to

$$
d_{1}\left(\mathbf{x}_{\mathbf{r}}, \mathbf{x} ; \omega\right)=\int G\left(\mathbf{x}_{\mathbf{s}}, \mathbf{x} ; \omega\right) d_{o}\left(\mathbf{x}_{\mathbf{s}}, \mathbf{x}_{r} ; \omega\right) d \mathbf{x}_{\mathbf{s}}
$$

in which, $\mathbf{x}$ denotes the position vector in the image and, when subscripted with $\mathrm{s}$ and $\mathrm{r}$, represent the position vector for source and receiver, respectively; $G$ is the Green function from the source to the image domain computed with the one-way wave equation, and $d_{1}$ is the extrapolated wavefield.
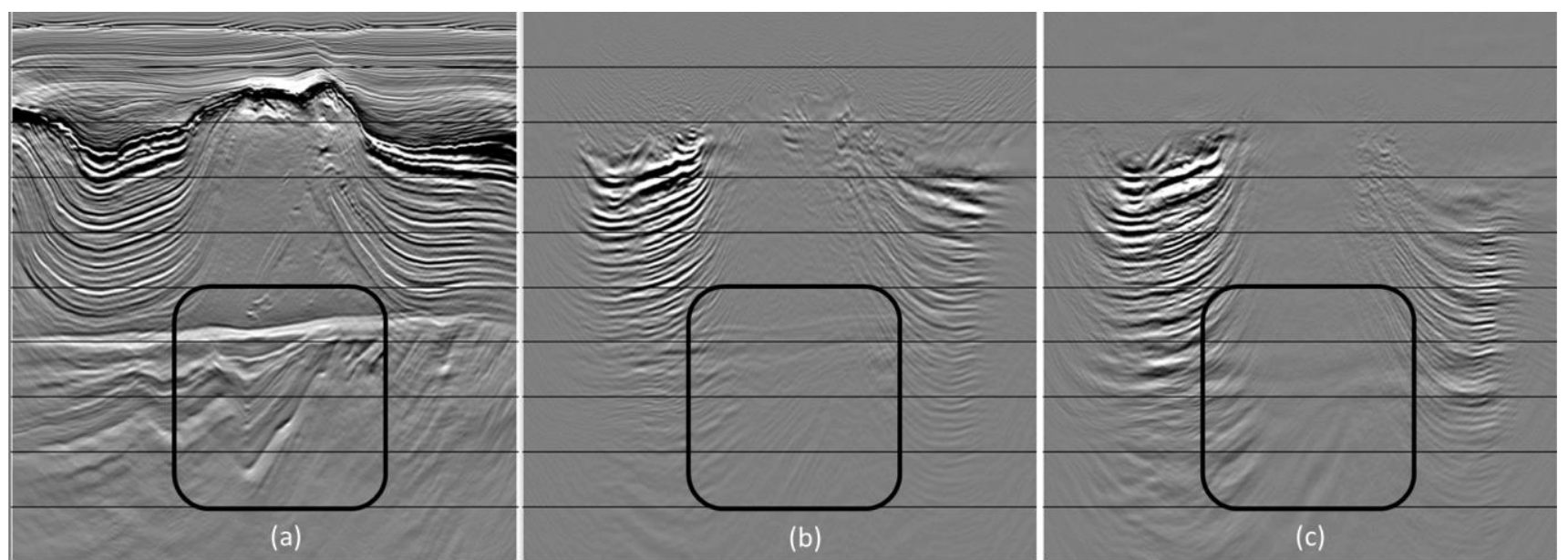

Figure 2 - Example of primary leakage in the interbed multiple modeling. (a) migration of the downgoing wavefield of the Lula Pilot nodes survey; (b) migration of downgoing interbed multiples generated at the top salt, with a not so deep enough muted input gathers; (c) migration of downgoing interbed multiples generated at the top salt, with adequately muted input gathers. Primary leakage has been almost completely mitigated in (c), compared to (b). 
In the sequence, wavefield $d_{1}$ is upward extrapolated, convolved with reflectivity $m^{\prime}$ (the reflectivity above the auxiliary horizon) at every depth step, and stored up to the minimum depth of the generator, using:

$$
d_{2}\left(\mathbf{x}_{\mathbf{r}}, \mathbf{x}^{\prime} ; \omega\right)=\int G\left(\mathbf{x}, \mathbf{x}^{\prime} ; \omega\right) d_{1}\left(\mathbf{x}, \mathbf{x}_{r} ; \omega\right) m^{\prime}(\mathbf{x}) d \mathbf{x},
$$

where $d_{2}$ is the extrapolated wavefield and the prime denotes the new image domain coordinates, which coincides with $\mathbf{x}$.

Then, wavefield $d_{2}$ is downward extrapolated down to the maximum depth, with injection and storage of the result for every depth, as stated by

$d_{3}\left(\mathbf{x}_{\mathbf{r}}, \mathbf{x}^{\prime \prime} ; \omega\right)=\int G\left(\mathbf{x}^{\prime}, \mathbf{x}^{\prime \prime} ; \omega\right) d_{2}\left(\mathbf{x}_{r}, \mathbf{x}^{\prime} ; \omega\right) d \mathbf{x}^{\prime}$

Finally, wavefield $d_{3}$ is upward extrapolated, with injection and convolution with reflectivity $m^{\prime \prime}$ (the reflectivity below the auxiliary horizon) for every depth, up to the acquisition surface, using:

$$
d_{m}\left(\mathbf{x}_{\mathbf{s}}, \mathbf{x}_{r} ; \omega\right)=\int G\left(\mathbf{x}^{\prime \prime}, \mathbf{x}_{\mathbf{s}} ; \omega\right) d_{3}\left(\mathbf{x}_{\mathbf{r}}, \mathbf{x}^{\prime \prime} ; \omega\right) m^{\prime \prime}\left(\mathbf{x}^{\prime \prime}\right) d \mathbf{x}^{\prime \prime},
$$

which yields the interbed multiples $d_{m}$ at the surface.

In the following, real data examples of the Santos Basin illustrate the method.

\section{Results}

Lula Pilot surveys were planned to investigate the 4D signal of the PreSalt reservoirs in the Lula field, Santos Basin, in response to a two-year WAG (water alternate gas) injection scheme (Kiyashchenko et al., 2020). Each survey comprises 970 nodes $325 \times 375 \mathrm{~m}$ apart in a hexagonal pattern and a shot carpet of approximately 150,000 shots $50 \mathrm{~m}$ apart. Top salt is by far the main generating reflector and, secondarily, the water-bottom also produces significant interbed multiples.

For a better multiple prediction, receiver gathers were interpolated to a grid $25 \times 25 \mathrm{~m}$. For all the examples shown in this paper the maximum temporal frequency was $60 \mathrm{~Hz}$, both for the modeling of multiples and for the one-way imaging. Since imaging upgoing data yields a poor image for shallower reflectors, it is fundamental to use the downgoing migrated image or any other image computed with the same velocity model as that of the multiple modeling as reflectivity.

In Figure 3, are shown the input downgoing data (on the left), the interbed modeled multiple for the water bottom (middle), and the interbed modeled multiple for the top salt (on the right) of a single shot line. Similarly, in Figure 4 one can see the same set of results for the upgoing data. Notice that in addition to lower amplitudes, water bottom interbed multiples show a chaotic aspect. These characteristics contribute for the lesser importance of these multiples in this area, compared to those of the top salt.

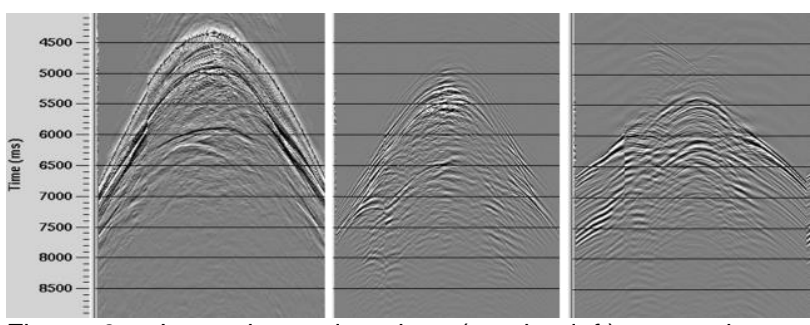

Figure 3 - Input downgoing data (on the left), water bottom interbed multiples (middle), and top salt interbed multiple (on the right).

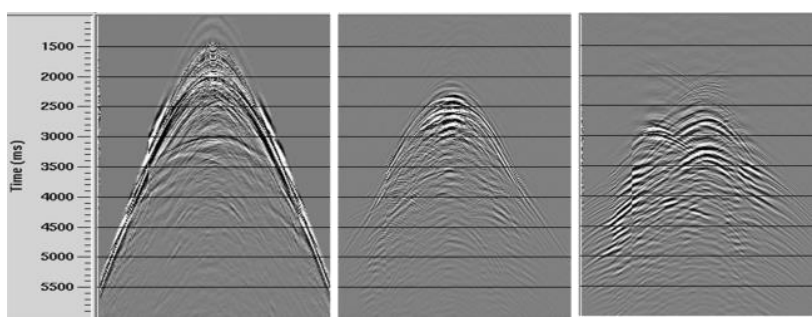

Figure 4 - Input upgoing data (on the left), water bottom interbed multiples (middle), and top salt interbed multiple (on the right).

Figure 5 shows one migrated inline of the downgoing data, in which the clear interference of the interbed multiples with the primary reflectors (black box in Figure $5 a$ ) is mimicked by the modeled and migrated interbed multiples (black boxes in Figures $b$ and $c$ ).
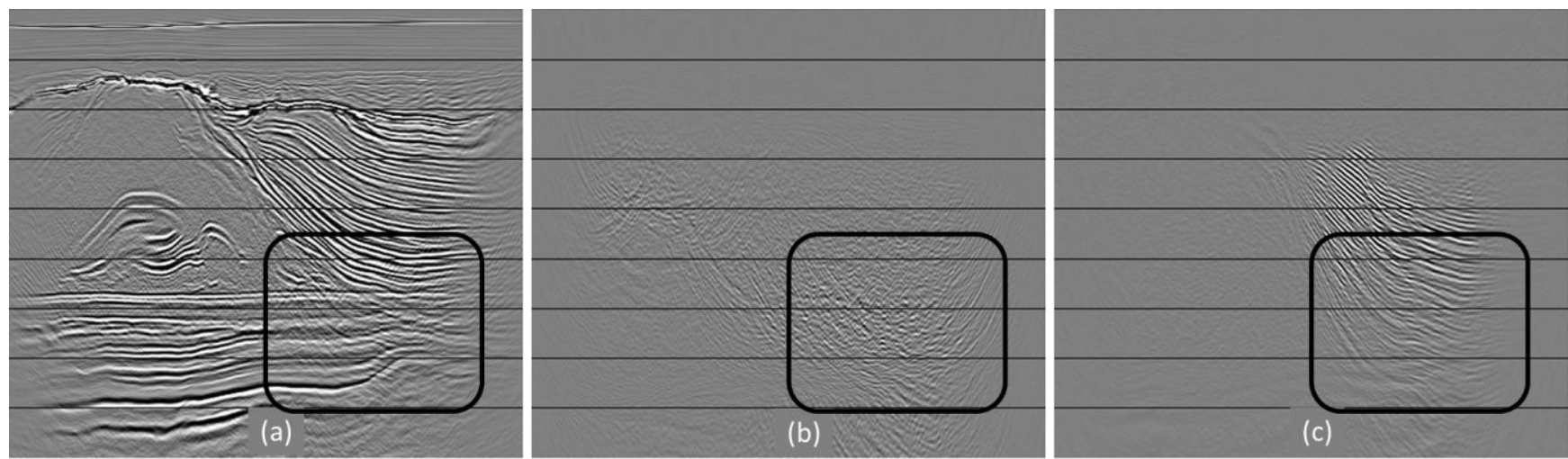

Figure 5 - Imaging of downgoing data: (a) input, (b) water bottom interbed multiples, and (c) top salt interbed multiples. The black box highlights the clear interference of the interbed multiples with the primary reflectors and its coincidence with the predicted multiples. 
Figure 6 shows the same inline as in the previous figure for the upgoing data, which is a bit noisier that the downgoing. Again, it is clear the coincidence between the modeled (black boxes in Figure $6 \mathrm{~b}$ and $\mathrm{c}$ ) and the interfering interbed multiples (black box in Figure 6a).

For the interbed multiple subtraction, we decided to use only those generated at the top salt since, in comparison, those generated at the water bottom are very low in amplitudes. Figure 7 shows the subtraction results for the downgoing (input at Figure 7a and output at $7 \mathrm{~b}$ ) and for the upgoing (input at Figure $7 c$ and output at $7 d$ ). Subtraction was performed in two passes, first in the crossline direction, then in the inline direction. The black boxes highlight the successful attenuation of the interbed multiples generated at the top salt. In addition, a horizon slice through the reservoir level is shown in Figure 8 and again the black boxes highlight the main differences. On the top is the downgoing data example (input at Figure 8a and output at Figure $8 \mathrm{~b}$ ) and at the bottom that for the upgoing (input at Figure $8 \mathrm{c}$ and output at Figure $8 \mathrm{~d}$ ). More sophisticated attenuation methods would, for sure, have yielded better results, especially concerning keeping primary energy.

\section{Conclusions}

Interbed multiples are persistent in nodes data in the Santos Basin. Its modeling and posterior attenuation are key for a successful PreSalt reservoir characterization and monitoring. This paper shows the promising adaptation of the Pica and Delmas method to nodes geometry, in both downgoing and upgoing modes. Prediction has no sampling limitation with respect to shot or receiver sampling as in data driven methods. However, the identification of a generator as well as the need of an accurate velocity model are mandatory.

\section{Acknowledgments}

We would like to acknowledge Petrobras for allowing publishing this work.
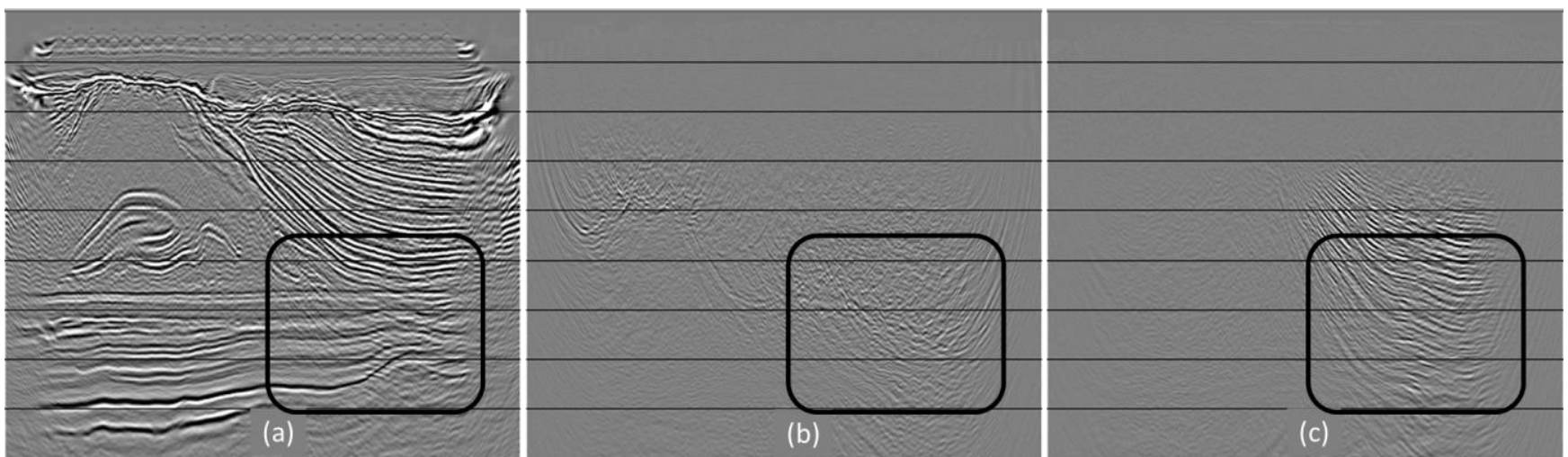

Figure 6 - Imaging of upgoing data: (a) input, (b) water bottom interbed multiples, and (c) top salt interbed multiples. The black box highlights the clear interference of the interbed multiples with the primary reflectors and its coincidence with the predicted multiples.

\section{References}

Cypriano L., Marpeau F., Brasil R., Welter G., Prigent H., Douma H., Velasques M., Boechat J., Carvalho P., Guerra C., Theodoro C., Martini A., and Cruz J.N., 2015, Improved imaging of pre-salt targets in the Santos basin off-shore Brazil through attenuation of inter-bed multiples. 14th International Congress of the Brazilian Geophysical Society \& EXPOGEF, Rio de Janeiro, Brazil: 1262-1267.

Kiyashchenko D., Wong W., Cherief D., Clarke D., Duan Y., and Hatchell P., 2020, Unlocking seismic monitoring of stiff reservoirs with 4D OBN: A case study from Brazil presalt. 90th Annual International Meeting, SEG, Expanded Abstracts, 3759-3763.

Hembd J., Griffiths M., Ting C.O., and Chazalnoel N., 2010, Application of interbed multiple attenuation in the Santos Basin, Brazil. SEG Technical Program Expanded Abstracts: 3451-3455.

Guerra C. and Hargreaves, N., 2019, Zero-offset internal multiple modeling in the image domain. 16th International Congress of the Brazilian Geophysical Society \& EXPOGEF, Rio de Janeiro, Brazil.

Krueger J., D. Donno, R. Pereira, D. Mondini, A. Souza, J. Espinoza, and A. Khalil, 2018, Internal multiple attenuation for four Pre-salt fields in the Santos Basin, Brazil. SEG Technical Program Expanded Abstracts: 4523-4527.

Pereira R., Ramzy M., Griscenco P., Huard B., Huang H., Cypriano L., and Khalil A., 2019, Internal multiple attenuation for OBN data with overburden/target separation: 89th Annual International Meeting, SEG, Expanded Abstracts, 4520-4524.

Pica, A. and Delmas, L., 2008, Wave-equation based internal multiple modeling in 3D: 78th Annual International Meeting, SEG, Expanded Abstracts, 2476-2480.

Staring M., Neut J., and Wapenaar K., 2018, Marchenko redatuming by adaptive double-focusing on $2 \mathrm{D}$ and $3 \mathrm{D}$ field data of the Santos basin. SEG Technical Program Expanded Abstracts: 5449-5453. 

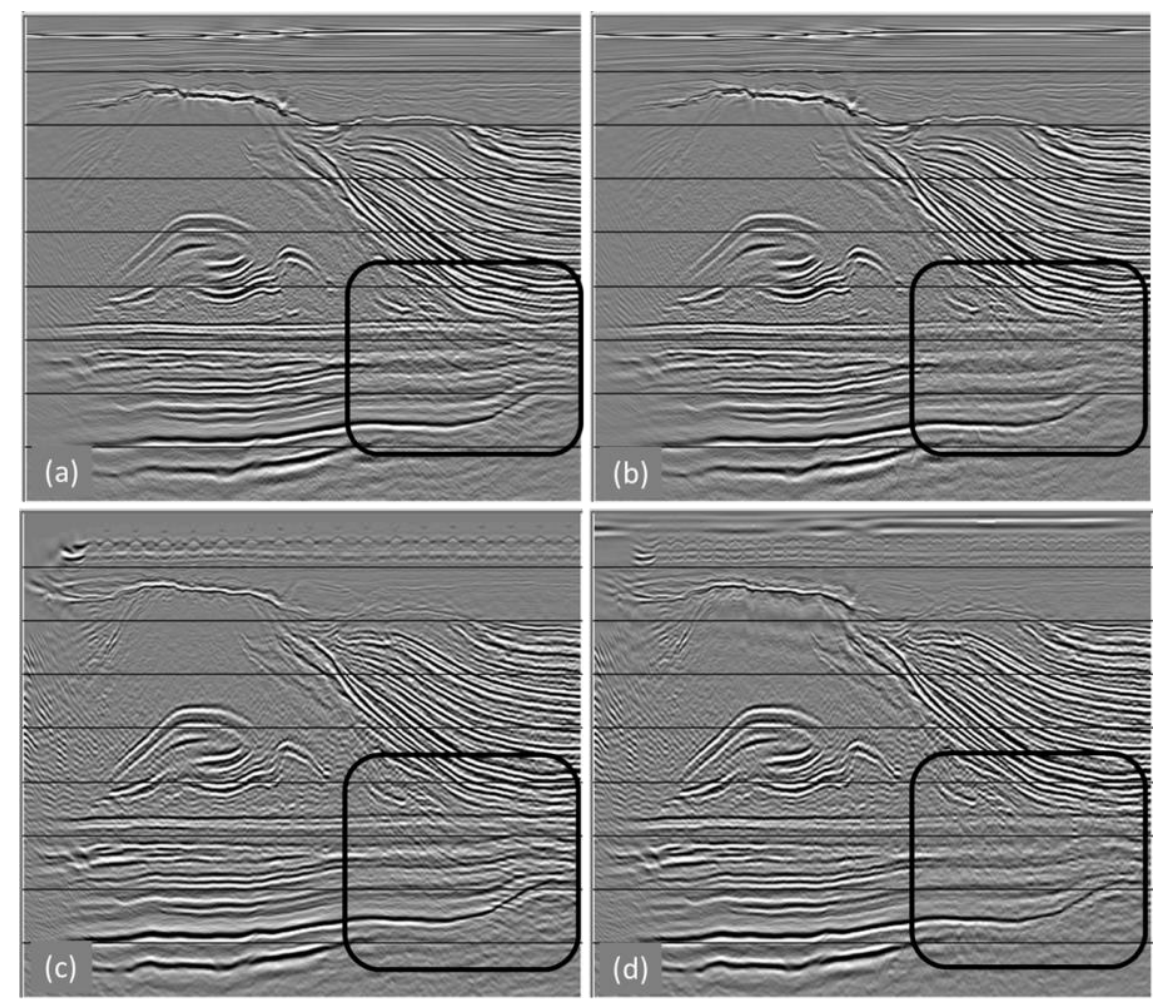

Figure 7 - Attenuation of the top salt interbed multiples: for the downgoing, (a) input and (b) output, and for the upgoing, (c) input and (d) output.
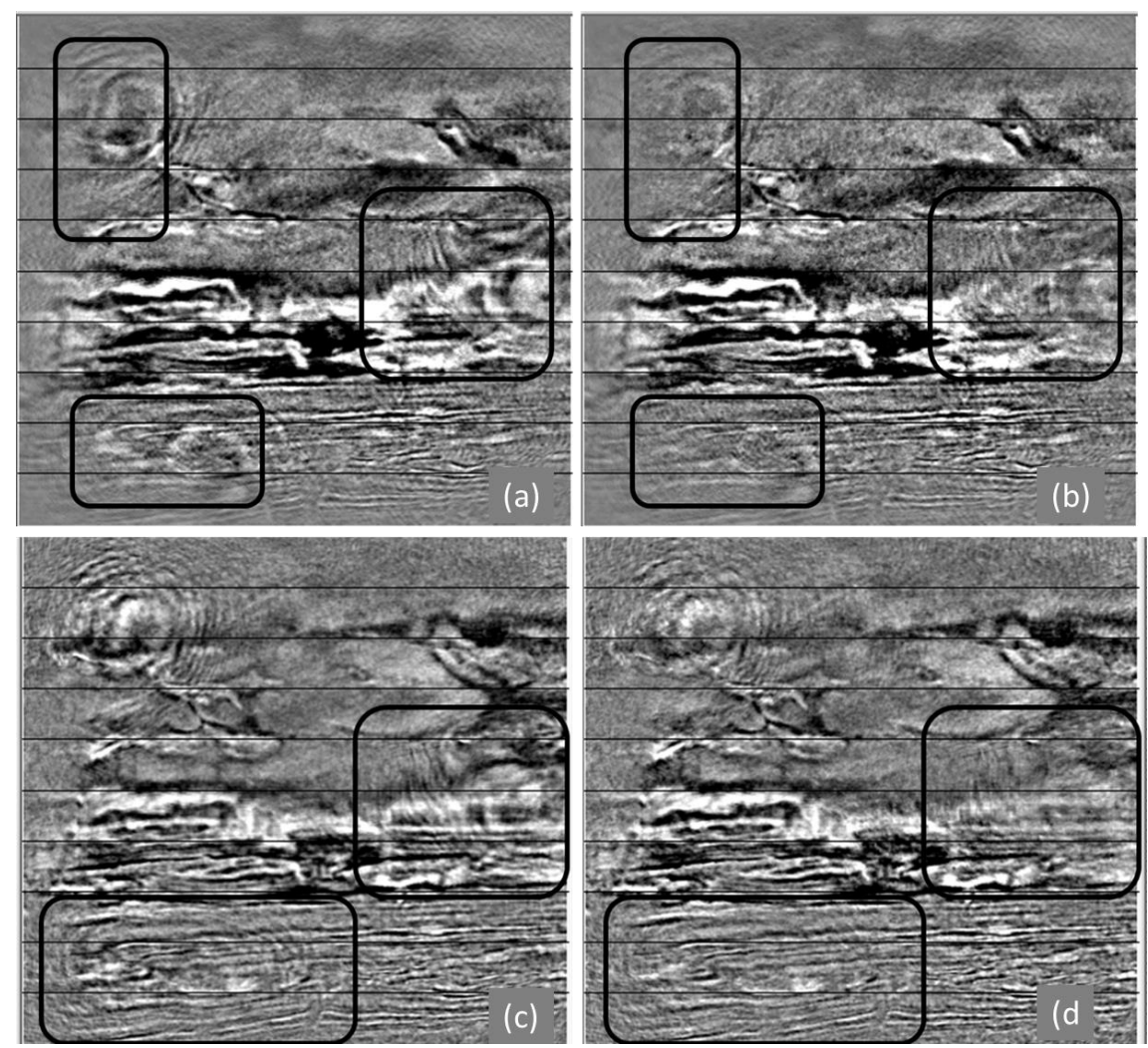

Figure 8 - Attenuation of the top salt generated interbed multiples illustrated in a horizon slice through the reservoir level: for the downgoing, (a) input and (b) output, and for the upgoing, (c) input and (d) output. 